\title{
Oral hygiene and ONJ: a review of the literature.
}

\author{
Paolo Boffano $^{1}$, ANNA MARIA AGNONE, Francesco Cavarra, ANDREA MELLE ${ }^{2}$, Vincenzo Rocchetti ${ }^{2}$, Tricarico \\ Gerardo $^{1}$ \\ 1 ASL VC \\ 2 University of Eastern Piedmont
}

Funding: The author(s) received no specific funding for this work.

Potential competing interests: The author(s) declared that no potential competing interests exist.

\section{Abstract}

The importance of oral hygiene to minimise their risk of ONJ has often been emphasized in the literature.

Some patients may require a change in behaviour in terms of brushing, interdental cleaning and other oral hygiene techniques, as well as other lifestyle behaviours such as diet and tobacco use. There may also be a benefit in prescribing high fluoride toothpaste for those patients with increased caries risk.

Therefore, dental hygienists and dental practitioners should give personalised preventive advice to help the patient optimise their oral health, emphasising the importance of having a healthy diet and reducing sugary snacks and drinks; maintaining excellent oral hygiene; and using fluoride toothpaste and fluoride mouthwash.

The aim of the present communication was to review the literature about the protocols, methods and advises about a correct home and professional oral hygiene in patients at risk for development of ONJ and in patients affected by ONJ.

Background: The importance of oral hygiene to minimise their risk of ONJ has often been emphasized in the literature. Some patients may require a change in behaviour in terms of brushing, interdental cleaning and other oral hygiene techniques, as well as other lifestyle behaviours such as diet and tobacco use. There may also be a benefit in prescribing high fluoride toothpaste for those patients with increased caries risk.

Therefore, dental hygienists and dental practitioners should give personalised preventive advice to help the patient optimise their oral health, emphasising the importance of having a healthy diet and reducing sugary snacks and drinks; maintaining excellent oral hygiene; and using fluoride toothpaste and fluoride mouthwash.

The aim of the present communication was to review the literature about the protocols, methods and advises about a correct home and professional oral hygiene in patients at risk for development of ONJ and in patients affected by ONJ. Materials and Methods: A review of the literature was performed using the MeSH terms ONJ; MRONJ; BRONJ; osteonecrosis; oral hygiene; professional oral hygiene.

Results: Patients with dental implants placed prior to commencement with anti-resorptive or antiangiogenic drugs, should be informed of the small risk of spontaneous MRONJ at those sites and should be provided information on how to minimise their risk.

Osteoporosis patients who consider dental implants during or after treatment with antiresorptive drugs should be informed 
of the risk of compromised bone healing and MRONJ following the procedure and the additional small risk of long-term implant failure.

Preventive dental regimes can decrease the risk of oral complications in patients that are starting antiresorptive therapy by reducing the need for subsequent extractions or other procedures which impact on bone. Some patients may require a change in behaviour in terms of brushing, interdental cleaning and other oral hygiene techniques, as well as other lifestyle behaviours such as diet and tobacco use. There may also be a benefit in prescribing high fluoride toothpaste for those patients with increased caries risk.

For patients who are about to commence treatment with anti-resorptive or anti-angiogenic drugs, or those who have very recently started drug therapy, oral hygienists should give personalised preventive advice to help the patient optimise their oral health, emphasising the importance of having a healthy diet and reducing sugary snacks and drinks; maintaining excellent oral hygiene; using fluoride toothpaste and fluoride mouthwash; stopping smoking; limiting alcohol intake; and regular dental checks.

Conclusions: The dentist and the oral hygienist should discuss with the patient the importance of improving and maintaining an effective dental hygiene, addressing other lifestyle factors such as diet, smoking status and alcohol consumption, and performing regular check-ups. 